\title{
1978 NOBEL PRIZE FOR PHYSICS Arno A. Penzias and Robert W. Wilson
}

(An account of the work of Peter Kapitza will be published in a future issue.)

After Sir Martin Ryle and Anthony Hewish, Nobel Prize-winners for Physics in 1974, the Nobel Committee honours again, together with P.L. Kapitza, two astronomers, and even more precisely two radioastronomers. Even if this decision, comes as a surprise to many physicists and astronomers, what is not a surprise, is the choice of the recipients: Arno Penzias and Robert Wilson. They are in effect very well known for their discovery of the background emission of the Universe, the famous $3 K$ (or rather $2.8 \mathrm{~K})$ blackbody radiation. This discovery is certainly one of the major steps in cosmology, comparable in importance to the discovery by Hubble of the expansion of the Universe.

A.A. Penzias was born in Munich in 1933; his family managed to escape nazism just before the war and found refuge first in England, then in the USA where Penzias studied at the Columbia University under C. Townes, another Nobel Prize-winner. He joined the Bell Telephone Laboratories in 1961 where he has worked since, partly in radioastronomy and partly in various fields of applied research in telecommunications. $\mathrm{He}$ is presently director of the research laboratory in radioastronomy, at the Crawford Hill Laboratories, Holmdel, New Jersey.

R.W. Wilson, born in Houston (Texas), is an alumnus of the California Institute of Technology where he got his $\mathrm{PhD}$ in Radioastronomy. $\mathrm{He}$ came to the Bell Telephone Laboratories in 1963, two years after Penzias. They have always worked together since and both signatures appear in an impressive number of scientific and technical papers. Wilson is now head of the radiophysics laboratory at the Crawford Hill Laboratories.

Their discovery of the blackbody radiation of the Universe is one of the most impressive achievements in experimental physics. In 1958, the Bell Lab physicist E.A. Ohm, making a calibration of a very special low noise antenna used for space telecommunications by reflexion over the Echo Satellite, noticed that it produced somewhat more noise than expected on the basis of the ground radiation picked up in the antenna sidelobes, and of the contribution of the antenna losses, the atmosphere and the
Galaxy. He, however, concluded that the errors in his measurements were larger than expected and did not worry about a possible extra signal.

Penzias and Wilson took over the problem and, after a careful investigation of all possible sources of noise and causes of error, concluded that this extra noise was indeed real. They further showed that it did not depend on the season and on the weather, was isotropic and unpolarized, and they assigned to it an extraterrestrial origin. The brightness corresponded to that of a blackbody at $3.5 \pm 1.0 \mathrm{~K}$ at the observing frequency of 4.08 $\mathrm{GHz}$. As the spectrum of the emission was not known, its true blackbody nature was not known either, and it has taken years since to establish it. In this very difficult type of absolute measurement, it is necessary first to separate in the total observed noise, the measured contribution of the noise of the receiver, then to measure or calculate and subtract from what is coming from the antenna, everything that is not the celestial contribution. This would not have been possible with enough certainty without the excellent quality of the Bell antenna, and also of the maser amplifier and accessories built by Penzias and Wilson themselves, helped by the technological background of their Company.

Some time after the discovery, in the Autumn of 1964, Penzias reported on it to R. Dicke, from Princetown University, a well-known cosmologist who is also an expert in radiometers. With his collaborators J. Peebles, R. Stokes and D. Wilkinson, Dicke had predicted the existence of a cosmological blackbody radiation at about $10 \mathrm{~K}$, and they were building a special radiometer to try to detect it. Doroshkevich and Novikov in the USSR had also had the same idea and decided that the $20-\mathrm{ft}$ horn reflector of the Bell Labs would be the ideal instrument to find the radiation. However Penzias and Wilson were before them, and Dicke and collaborators and themselves published, one after the other, two letters to the Astrophysical Journal in the 1 July, 1965 issue (142, 414 and 419 ), one theoretical and the other observational. Dicke's idea was that the observed radiation is the re-

\section{J. Lequeux, Meudon \\ (Paris Observatory)}

lict of the intense and very hot radiation filling the early universe in the big bang models. At a temperature less than $10^{10} \mathrm{~K}$, equilibrium between radiation and matter through pairproduction and annihilation ceased and the radiation evolved with little coupling with matter: during the expansion of the Universe, its shape remained that of blackbody radiation with a temperature decreasing like the inverse of the characteristic dimensions of the Universe. It turns out that Dicke was not actually the first to have had this idea. Apparently this was George Gamov, who wrote to Penzias after the discovery was publicized: "Thank you for sending me your paper on $3^{\circ} \mathrm{K}$ radiation. It is very nicely written except that "early history" is not "quite complete". The theory of, what is now known as, "primeval fireball" was first developed by me in 1946 (Phys Rev., 70 (1946) 572; 74 (1948) 505; Nature, 162 (1948) $620)$. The prediction of the numerical value of the present (residual) temperature is to be found in Alpher and Hermann's paper (Phys. Rev. 75 (1949) 1093 who estimated it as $5^{\circ} \mathrm{K}$. Alpher and Hermann were Dicke's students; other estimates from this group fel between $5^{\circ}$ and $28^{\circ}$ on the basis of assumptions (that) today (would be regarded as) mostly questionable."

In fact, the blackbody radiation could have been found quite a long time before Penzias and Wilson, in an indirect way. It was known since the works of Adams and of McKellar in 1941, that the two lower rotational levels of the $\mathrm{CN}$ interstellar molecule have a relative population corresponding to an excitation (rotation) temperature of about $3 \mathrm{~K}$. We now realize that these levels are in equilibrium with the universal radiation at the wavelength of the corresponding rotational transition, $2.64 \mathrm{~mm}$ but no one had been able to imagine that before the discovery of Penzias and Wilson.

The cosmological significance of the discovery is outstanding. It seems impossible to explain, in the framework of the steady state theory advocated by Hoyle, Gold, Bondi and others, why the Universe should be filled with blackbody radiation. This theory thus received a mortal blow (it had already suffered very much when 
the Cambridge radioastronomers discovered the evolution of extragalactic radiosources). The efforts of promotors of this theory to try to simulate the $3 \mathrm{~K}$ blackbody radiation by the superposition of the emission of many unresolved extragalactic sources failed, and this was the end of the steady state. Moreover, as realized already by Gamov, it is possible when the present temperature of the Universe is known, to know something of the early Universe. It is even possible to determine, in the framework of the usual assumptions (isotropy, uniformity, General Relativity, zero cosmological constant) whether the Universe is open or closed, provided that one knows another fundamental quantity, the present density or the abundance of elements like helium or deuterium formed in the big bang. Although none of these quantities is known with sufficient unambiguity, there is a widespread feeling amongst astronomers that the Universe is open and is expanding for ever.

At present, astronomers are working on the blackbody radiation in three different directions: deviations from Planck's law, large-scale anisotropies and clumpiness. Detection of these effects would be of great importance for cosmology for reasons which are too long to be discussed here. However, although there are hints for short wavelength deviations from Planck's law and for a large scale anisotropy, neither of these two effects is established with complete certainty.

After the success of Penzias and Wilson, they were allowed by the Bell Labs to develop a group of radioastronomy. This group was the first to build and use a really good receiver of millimeter waves, and it was to open a new era in astronomy. With the receiver at the focus of the $11 \mathrm{~m}$ millimeter-wave radiotelescope of the $\mathrm{Na}$ tional Radio Astronomy Observatory at Kitt Peak (Arizona), they discovered eight interstellar molecules in a few days, including the ubiquitous CO. This was in 1970; previously, only seven interstellar molecules were known, three optically, and the rest in radio thanks initially to the perseverance of $\mathrm{Ch}$. Townes. At present, eight years after, we count nearly 50 interstellar molecules, many with several isotopic substitutions. A large number of groups are working in this field and building new instruments (in particular the French-German Institute for Radio Astronomy in the Millimeter range, IRAM, whose official birth is expected soon). The Bell Labs group is still at the forefront of this

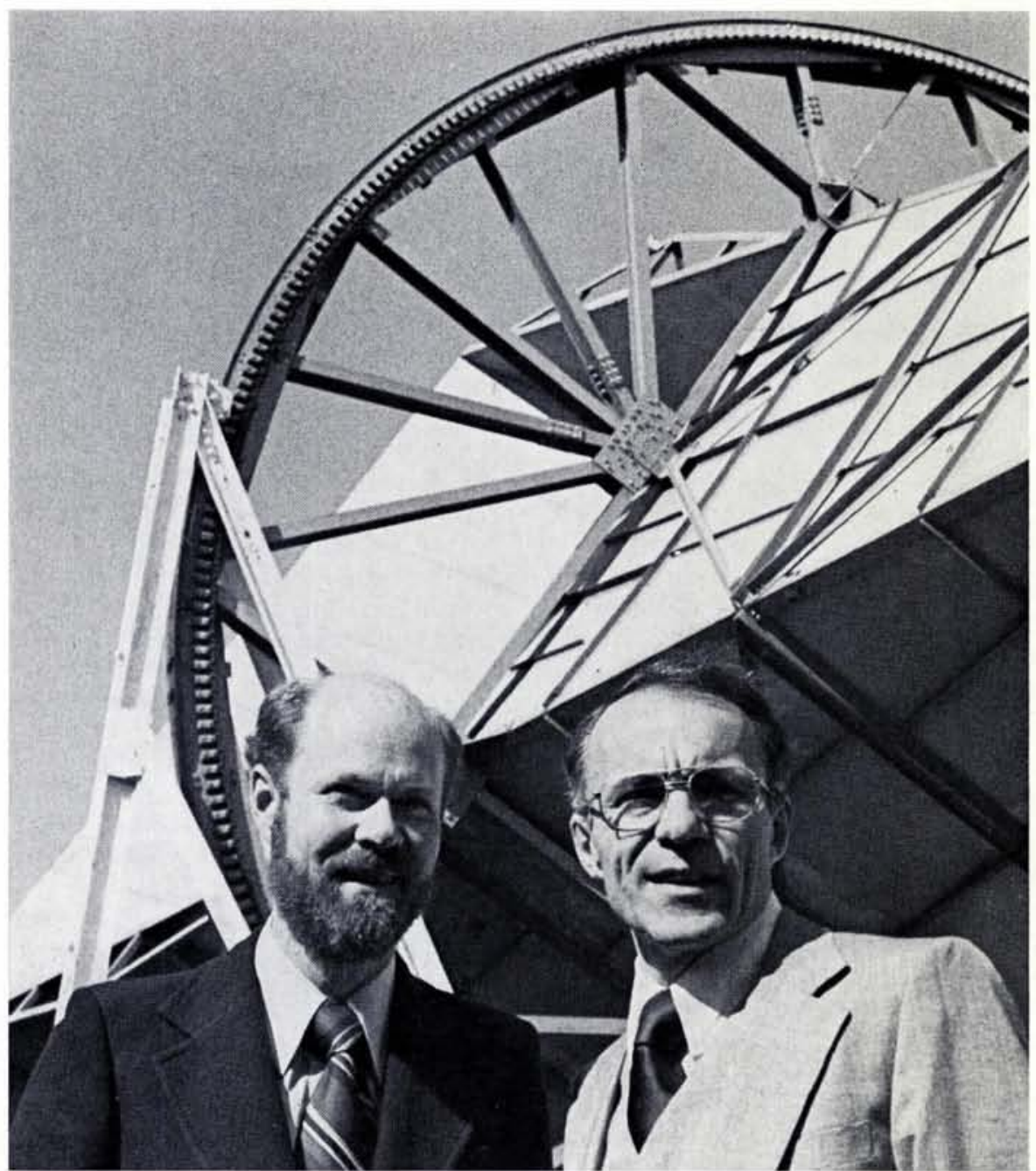

Robert Wilson (left) and Arno Penzias (right) posing in front of the 20ft horn antenna of the Bell Telephone Laboratories with which they discovered the blackbody radiation of the Universe.

research, thanks to excellent third generation receivers and a new high quality millimeter wave antenna completed in 1977. Penzias and Wilson and their students specialize in observations of isotopic substitutions of interstellar molecules, with the hope of finding galactic gradients of abundance ratios of isotopes of different nucleosynthetic origin, like $H$ and $D$, ${ }^{12} \mathrm{C}$ and ${ }^{13} \mathrm{C},{ }^{14} \mathrm{~N}$ and ${ }^{15} \mathrm{~N}$, etc. Such gradients have indeed been detected by themeselves and other astrono- mers, but the interpretation of the data is not straightforward. They are nevertheless of fundamental importance for understanding the evolution of galaxies as well as for cosmology.

The community of astronomers, and more especially of radioastronomers, in Europe and elsewhere, is extremely happy and without reservations with the choice of the Nobel Committee. This choice honours not only two great astronomers, but also two superb experimental physicists.

\section{FU/BERLIN \\ FREIE UNIVERSITATT BERLIN - FACHBEREICH PHYSIK}

Applications are invited for a

\section{Full Professorship in Biophysics / Medical Physics}

The appointee is expected to perform research in a field of biophysics or medical physics and to participate in the teaching duties of the Department with particular emphasis on biomedical physics. Applications should be submitted by 10 March, 1979, to the:

Physics Department,

Freie Universität Berlin,

Hüningerstr. 44 / 1000 Berlin 33. 